\title{
Oligomeric and fibrillar amyloid-beta42 studied by cryo-TEM
}

\author{
J.S.Wu*\#, M.P. Lambert**, P.T. Velasco**, S. Gutiontov*, S. Sharma*, H.M. Joshi*, G. \\ Shekhawat*, W.L. Klein** and V.P. Dravid*\# \\ *Department of Materials Science and Engineering, and \#NUANCE Center, Northwestern \\ University, Evanston, IL, 60208, USA. \\ **Department of Neurobiology and Physiology, Northwestern University, Evanston, IL 60208, USA
}

For more than 20 years, amyloid beta $(A \beta)$-derived fibrillar peptides were considered the primary toxin in Alzheimer's disease (AD) pathology, even though the abundance of A $\beta$ fibrillar plaques was shown to be a poor pathological correlate of dementia. Recently, however, an alternative to the disease-defining $\mathrm{A} \beta$ fibrils has emerged in which $\mathrm{AD}$ toxicity derives from neurologically active $\mathrm{A} \beta$ oligomers (also called ADDLs, for amyloid beta-derived diffusible ligands) [1]. ADDLs bind to mature hippocampal neurons in a ligand-like manner, stimulate tau phosphorylation, and cause other AD-related pathology [2]. ADDLs form the core of a novel unifying mechanism for AD pathogenesis, explaining why early stage disease is specific for memory loss and accounting for major facets of AD neuropathology.

Since $A \beta$ peptides self-aggregate, the structures of the various aggregated species have been a matter of intense interest. ADDL fractionation experiments show the ligand activity requires molecules greater in mass than $50 \mathrm{kDa}[3]$. This size requirement is consistent with the positive identification of $A \beta 12$ mers as the causative agent of memory failure. In the current work, ADDLs, fibrils, and ADDLs fractionated using a $50 \mathrm{kDa}$ MicroCon filter were examined using Cryo-TEM. Samples were prepared by a Gatan plunger system and transferred into a Hitachi HF-8100 electron microscope for observation by using a Gatan cryo-transfer system. Figure 1 shows the several micron long fibers of fibrillar amyloid that have diameters between 10-12 nm, dimensions that agree with results obtained by negative-stain electron microscopy [4]. It should be noted the many spherical particles are enmeshed in the fiber network. In Figure 2, ADDLs $>50 \mathrm{kDa}$ (retentate) are imaged. Contrary to results found with liquid and dry atomic force microscopy, in which only spherical structures in the low nm range are found, a high density of fibrils is present. The reason for this discrepancy is presently under further scrutiny. Figure 3 shows an image of ADDLs $<50$ $\mathrm{kDa}$ in which very few fibrils and a high density of spherical particles with different diameters are seen. Cryo-TEM is thus shown to be a promising tool for the characterization of the native and pristine morphology of amyloid beta peptide species, which otherwise may get perturbed due to surface interactions during SPM observations or staining effects in conventional stained specimens in EM.

References

[1] M.P. Lambert, et al., PNAS 95 (1998) 6448.

[2] W.L. Klein, et al., Why Alzheimer's is a disease of memory, in D.J. Selkoe, et al. (ed.) Synaptic Plasticity and the Mechanism of Alzheimer's disease, Ipsen Foundation, Springer, Berlin, Germany, (2008) 103-132.

[3] P.N. Lacor, et al., J. Neurosci. 24 (2004) 10191.

[4] A.S. Cohen, et al., Electron Microscopy of amyloid. In J.R. Harris, (ed.) Electron Microscopy of Proteins, vol. 3. Academic Press, London, UK, (1982) 165-205.

[5] This work was performed in the EPIC facility of NUANCE Center at Northwestern University. The research is supported by NIH-CCNE and NSF-MWN programs. 


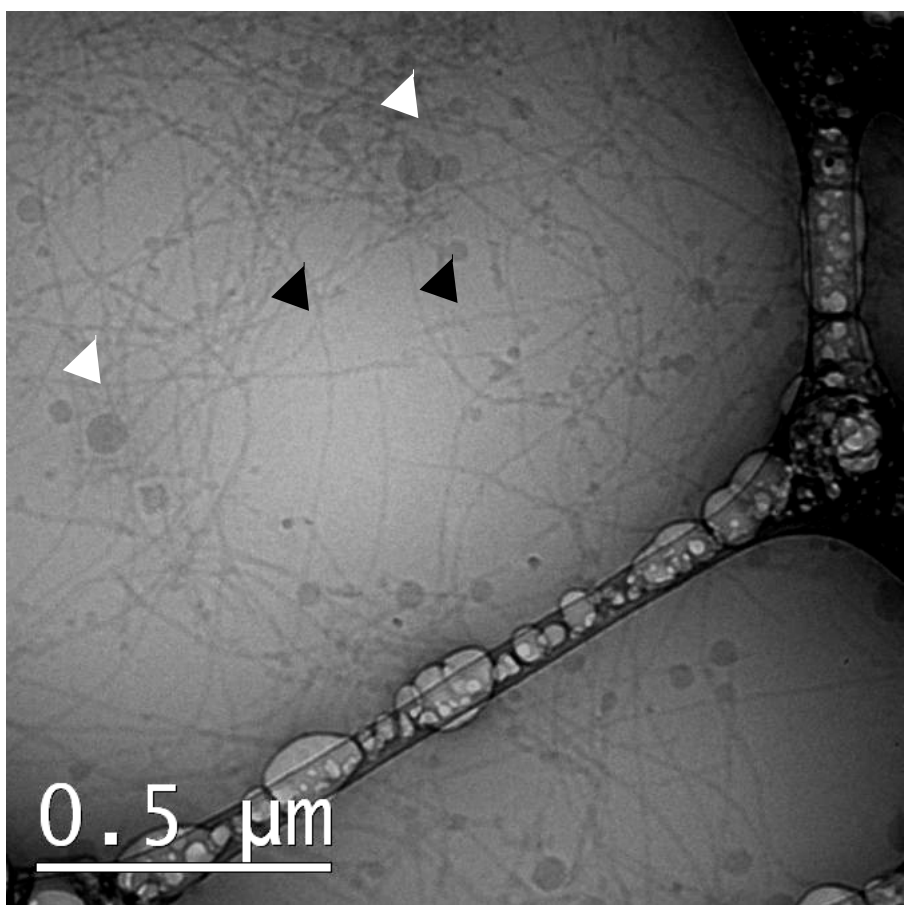

Figure 1. Cryo-TEM image of Fibrillar amyloid. 1wo tibers with diameter of $10-12 \mathrm{~nm}$ are indicated y black arrowheads, while two round particles are

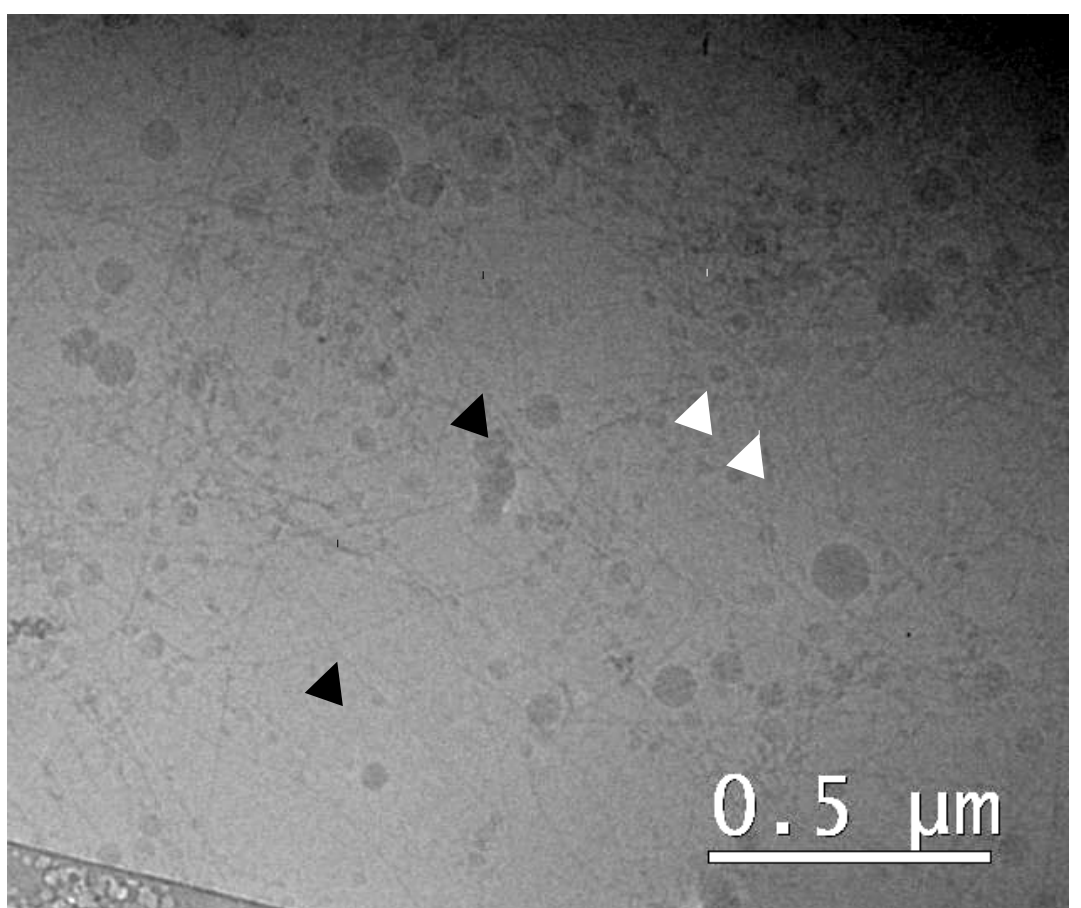

Figure 2. Cryo-TEM image of retentate which was left o the filter when ADDLs solution was filtered. Two fibrils with diameter of 5-7 nm are indicated by blac arrowheads, while two round particles with diameter o about $20 \mathrm{~nm}$ are indicated by white arrowheads.

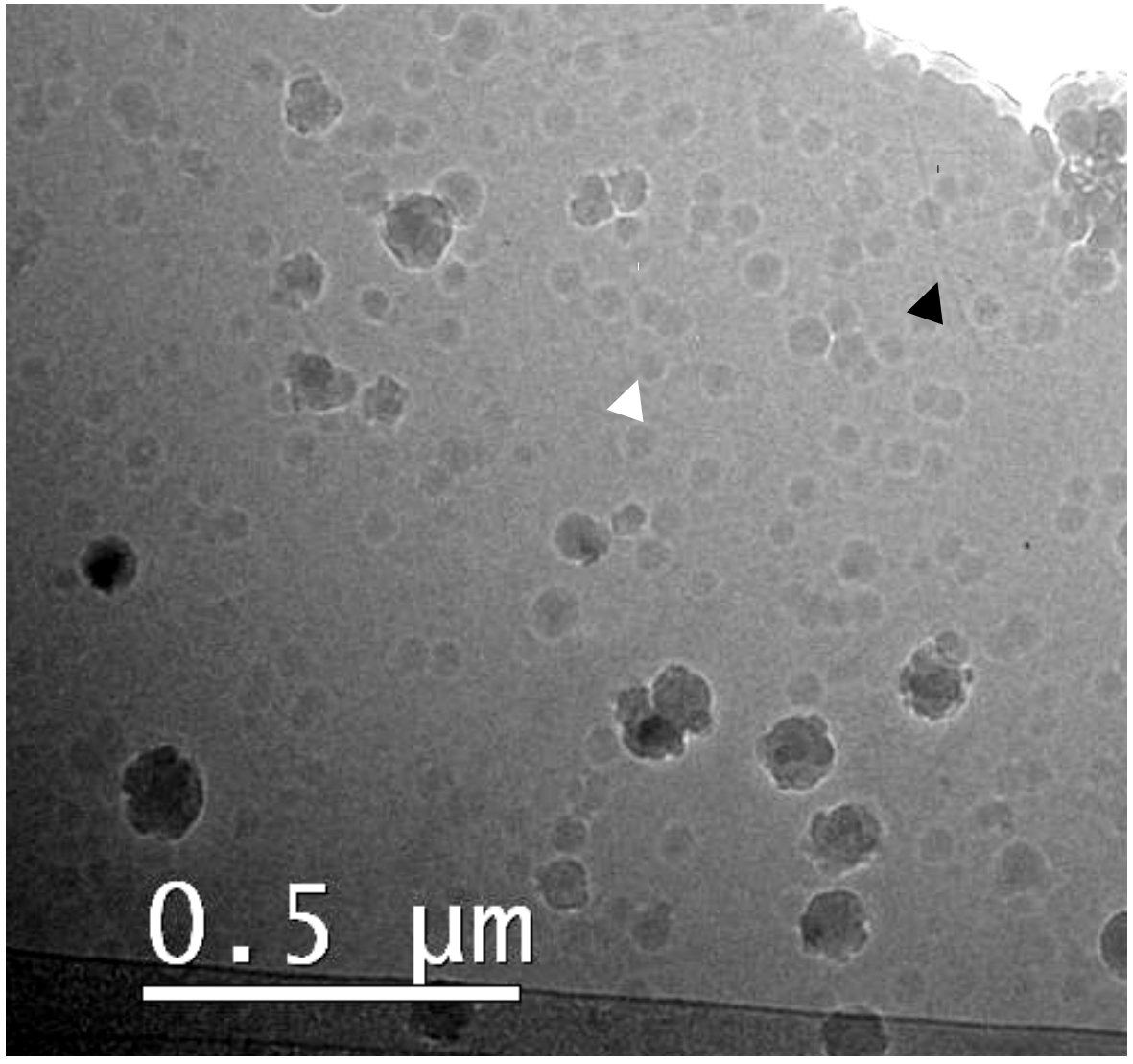

Figure 3. Cryo-TEM image of filtrate which went through the filter whe ADDLs solution was filtered. A fibril is indicated by blac arrowheads, while one round particle is indicated by white arrowheads. 